\title{
Equity aspects of the Primary Health Care Choice Reform in Sweden - a scoping review
}

Bo Burström ${ }^{1 *}$, Kristina Burström,2, Gunnar Nilsson ${ }^{3}$, Göran Tomson ${ }^{4}$, Margaret Whitehead ${ }^{1,5}$ and Ulrika Winblad ${ }^{6}$

\begin{abstract}
Background: Good health and equal health care are the cornerstones of the Swedish Health and Medical Service Act. Recent studies show that the average level of health, measured as longevity, improves in Sweden, however, social inequalities in health remain a major issue. An important issue is how health care services can contribute to reducing inequalities in health, and the impact of a recent Primary Health Care (PHC) Choice Reform in this respect. This paper presents the findings of a review of the existing evidence on impacts of these reforms.

Methods: We reviewed the published accounts (reports and scientific articles) which reported on the impact of the Swedish PHC Choice Reform of 2010 and changes in reimbursement systems, using Donabedian's framework for assessing quality of care in terms of structure, process and outcomes.

Results: Since 2010, over 270 new private PHC practices operating for profit have been established throughout the country. One study found that the new establishments had primarily located in the largest cities and urban areas, in socioeconomically more advantaged populations. Another study, adjusting for socioeconomic composition found minor differences. The number of visits to PHC doctors has increased, more so among those with lesser needs of health care. The reform has had a negative impact on the provision of services for persons with complex needs. Opinions of doctors and staff in PHC are mixed, many state that persons with lesser needs are prioritized. Patient satisfaction is largely unchanged. The impact of PHC on population health may be reduced.

Conclusions: The PHC Choice Reform increased the average number of visits, but particularly among those in more affluent groups and with lower health care needs, and has made integrated care for those with complex needs more difficult. Resource allocation to PHC has become more dependent on provider location, patient choice and demand, and less on need of care. On the available evidence, the PHC Choice Reform may have damaged equity of primary health care provision, contrary to the tenets of the Swedish Health and Medical Service Act. This situation needs to be carefully monitored.
\end{abstract}

Keywords: Equity, Inequalities, Health care need, Primary Health Care Choice Reform, Quality of care, Reimbursement system, Resource allocation

\footnotetext{
* Correspondence: bo.burstrom@ki.se

${ }^{1}$ Department of Public Health Sciences, Equity and Health Policy Research

Group, Karolinska Institutet, SE 17177 Stockholm, Sweden

Full list of author information is available at the end of the article
} 


\section{Background}

In many countries, including Sweden, primary health care $(\mathrm{PHC})$ is the basis of the health care system and contributes in an important way to the improvement of health in the population [1]. Good health and equal health care are the cornerstones of the Swedish Health and Medical Service Act [2]. Recent studies show that the average level of health, measured as longevity, improves in Sweden, among men and women [3]. However, social inequalities in health remain a major issue, new health divides are surfacing and the Government has recently commissioned an investigation into how inequalities in health can be reduced [4]. An important issue is how health care services can contribute to reducing inequalities in health, and the impact of recent reforms in $\mathrm{PHC}$ in this respect. This paper presents the findings of a review of the existing evidence on impacts of these reforms, as well as identifying the gaps in the current literature that need to be addressed by new empirical studies.

\section{The Swedish welfare state and health care system}

Swedish welfare services, including health care services, were developed after World War II, with the aim of creating a comprehensive public system for provision of services, of high quality and universally accessible for all $[5,6]$. The notion of providing good quality care services to the whole population has been regarded as part of the public welfare system in Sweden, providing high quality health care, schools, elder care and other social services to the entire population [5]. A universal welfare system, such as the Swedish health care system, requires the loyalty of the middle class in order to gain legitimacy and be sustained [7]. This in turn means that the services have to be of high quality to satisfy all users, an idea referred to by Rothstein [8] as "the high-quality standardized solution". If successful, the system could then as intended promote egalitarianism and social integration.

The Swedish health care system is tax funded and the responsibility for it is decentralized to county councils and regions, which collect taxes for the purpose [5]. As other parts of the welfare system, the health care system is based to a great extent on egalitarian principles, and the national level provides legislation and guidelines for health care. The main objective for Swedish health care, as expressed in the Health and Medical Services Act [2] from 1982, is good health in the entire population and health on equal terms, and equitable care based on need. The bulk of health services have been operated by public providers, but in recent years the proportion of private (for-profit) providers has increased, particularly in outpatient care and $\mathrm{PHC}$ [5].

The core function of Swedish PHC is to be the first health care contact for the population, to provide holistic and comprehensive care, ranging from health promotion, disease prevention and curative care to rehabilitation. PHC deals with the whole population, the whole individual, the entire disease panorama, and the disease process over the entire life course of individuals. This distinguishes PHC from secondary and tertiary care, which provide important services at specific occasions of patients' diseases.

Public PHC centres in Sweden have been established in a planned manner in local residential catchment areas to serve the population, and typically include 4-10 general practitioners (GPs), nurses, other paramedical professionals (physiotherapists, occupational therapists, podiatrists). In addition many $\mathrm{PHC}$ centres provide maternal and child health services. PHC also includes outreach services, not least through district nurses, to patients who need such services, and interacts with other public authorities [5]. PHC also offers on-site medical care services to residents in local nursing homes. Although not a gate keeper to secondary care as in some other countries, PHC is the first point of contact for most patients in Sweden who end up in secondary care [5].

The proportion of GPs and level of resources going to $\mathrm{PHC}$ in Sweden is lower compared to other high-income countries [9]. However, in a recent Swedish government investigation, a well-functioning PHC was mentioned as "probably the single most important activity by which the health care system can contribute to improve equity in health" ([10] p 375).

In international comparison, the average number of outpatient visits (to PHC as well as to specialist outpatient services) is considerably lower in Sweden than in other countries. In Sweden (and Finland) the average number of visits is about three per person and year, compared to about six for OECD countries [9]. This may be due to the organization of health care in Sweden, where a greater number of visits are done by nurses, and to the fact that Swedish health care is dominated by hospital based care. Nevertheless, access to outpatient care is considered to be lower in Sweden than in other countries.

\section{Choice reform and market orientation in primary health care}

In recent years market orientation has increased in the Swedish health care system as a whole. A national law on freedom of choice by citizens was passed in 2008 [11], to enable citizens to choose among providers in different sectors, including health and social care [12]. In 2010 an amendment was made to the Health and Medical Services Act, mandating the regions and county councils to allow citizens to choose their PHC provider, and to allow private providers of $\mathrm{PHC}$ to freely establish practices, if they met certain defined criteria. The 
objectives of this PHC Choice Reform were to increase patient choice, expand the provision of private health care to increase access to care, and to increase quality and innovation through competition among providers [11-13].

The law on freedom of choice was enforced by a centre-right government and there have been differing ideological views on the benefits of the PHC Choice Reform. The PHC Choice Reform implies a shift from an egalitarian towards a libertarian ideology in health care [14]. An analysis of policymakers' arguments when the PHC Choice Reform was legislated focused on whether and how the PHC Choice Reform harmonizes with the emphasis on equity in the Health and Medical Services Act, which population groups will actually benefit from the reform, and ultimately how the reform may impact on the role of $\mathrm{PHC}$ on population health and inequalities in health [15]. The study concluded that because health inequalities is one of the main challenges, the impact of health care reforms on equity should receive more attention in policy making [15].

Theoretically, the reform may impact in different ways on equity aspects of $\mathrm{PHC}$. On the one hand, access to care may be increased for all by allowing free establishment of providers; on the other hand, the free establishment may result in providers choosing where to establish, and reduce political opportunities for deliberate need-based resource allocation between PHC centres. In addition, the Choice Reform may impact on the role and assignment of PHC and thereby the prioritization of patients and the work of GPs. The organization of work and prioritization of patients in $\mathrm{PHC}$ is further affected by the type of financial reimbursement system employed, and how different PHC activities are incentivized. The reform has also subsequently been implemented differently in different county councils [14]. However, there is little scientific evidence on the impact of the reform.

Donabedian's framework for assessing quality of care [16], which distinguishes three aspects of quality in care: structure, process and outcomes, may be used as a point of departure for the analysis. In Donabedian's framework, structure refers to the settings in which care occurs, including facilities, equipment and monetary resources, human resources and organizational structure such as staff organization and methods of reimbursement. Process describes what is done in health care, including the patient's seeking care as well as the practitioner's activities in diagnosing and treating the patient. Outcome denotes the effects of care on health status of patients and populations, also including the patient's satisfaction of care [16].

In view of the emphasis on equity in Swedish health care policy, one important research question is how the PHC Choice Reform and increased market orientation will affect $\mathrm{PHC}$ in terms of equity aspects on structure, process and outcome of PHC. In particular, the reform may influence: 1) The establishment of new PHC clinics and resource allocation between PHC clinics, 2) The organization and implementation of $\mathrm{PHC}$ and how different patient groups are prioritized, and 3) The impact of PHC on population health.

The aim of this study was to review and analyze the evidence regarding the equity impact of the PHC Choice Reform in Sweden.

\section{Methods}

We did a scoping review of the published accounts (scientific articles and reports) which reported on the impact of the Swedish PHC Choice Reform of 2010 and changes in reimbursement systems, from 2008 to September 2016. From a search on PubMed and Web of Science we found six scientific articles. We also searched for "grey literature" including publications from relevant public agencies in Sweden concerning the PHC Choice Reform and found nine publications, three with a nationwide focus (one with in-depth analysis of data from three county councils), two covering three county councils and four reports were based solely on data from Stockholm County Council where the reform was introduced already in 2008, before it was legislated nationally in 2010 .

The results of the review are organized according to Donabedian's framework of structure, process and outcome. The results are summarized in a narrative manner.

\section{Results}

The review resulted in 6 scientific articles and 9 reports, which are presented in Table 1 . The main objectives of the PHC Choice Reform were to increase patients' choice of $\mathrm{PHC}$ provider, expand the provision of privately provided health care and increase quality and innovation through competition among providers [12]. An overview of the effects of the PHC Choice Reform based on Donabedian's framework and different reimbursement systems is presented in Table 2 .

\section{Effects on the structure of PHC - new facilities and reimbursement \\ Effects on the establishment of PHC facilities}

Since 2010, over 270 new private PHC practices have been established throughout the country, operating for profit [17]. In 2014 the Swedish National Audit Office presented an investigation of the PHC Choice Reform [18]. Their report concluded that the number of PHC clinics had increased in 20 out of 21 county councils, but that the new establishments had primarily located in the largest cities and urban areas, in socioeconomically more advantaged populations [18]. 
Table 1 List of reviewed publications

\begin{tabular}{|c|c|c|c|c|c|c|}
\hline Publication & $\begin{array}{l}\text { Ref. } \\
\text { no. }\end{array}$ & Year & Area(s) & Data & Focus & Results \\
\hline \multicolumn{7}{|l|}{ Scientific articles } \\
\hline Beckman, Anell & {$[23]$} & 2013 & Region Skåne & $\begin{array}{l}\text { Population register } \\
\text { data }\end{array}$ & $\begin{array}{l}\text { Process of care - } \\
\text { PHC visits }\end{array}$ & $\begin{array}{l}\text { Visits increased more among high-income than } \\
\text { low income earners }\end{array}$ \\
\hline Agerholm et al & {$[25]$} & 2015 & $\begin{array}{l}\text { Stockholm County } \\
\text { council }\end{array}$ & $\begin{array}{l}\text { Population register } \\
\text { data, public health } \\
\text { survey data }\end{array}$ & $\begin{array}{l}\text { Process of care - } \\
\text { PHC visits }\end{array}$ & $\begin{array}{l}\text { Visits increased more among person with } \\
\text { lesser needs; less among those with greater } \\
\text { needs }\end{array}$ \\
\hline Glenngård & [32] & 2013 & $\begin{array}{l}\text { Region Halland, } \\
\text { Skåne, Västra } \\
\text { Götaland }\end{array}$ & Patient survey data & $\begin{array}{l}\text { Outcome - Patient } \\
\text { satisfaction }\end{array}$ & $\begin{array}{l}\text { Satisfaction with primary care higher in areas } \\
\text { with low level of social deprivation and in } \\
\text { smaller practices }\end{array}$ \\
\hline Maun et al & {$[27]$} & 2013 & Gothenburg & $\begin{array}{l}\text { Interviews with } 24 \\
\text { PHC managers }\end{array}$ & $\begin{array}{l}\text { Process of care - } \\
\text { doctors' views }\end{array}$ & $\begin{array}{l}\text { Prioritisation conflicts among doctors between } \\
\text { patients with different needs and demands. } \\
\text { Chronically ill patients were crowded out. }\end{array}$ \\
\hline Hollman et al & {$[28]$} & 2014 & Gothenburg & $\begin{array}{l}\text { Interviews with PHC } \\
\text { district nurses }\end{array}$ & $\begin{array}{l}\text { Process of care - } \\
\text { nurses' views }\end{array}$ & $\begin{array}{l}\text { Reimbursement system emphasizes doctors and } \\
\text { plays down nurses' role. Negative for job satisfaction } \\
\text { and work environment }\end{array}$ \\
\hline Isaksson et al & {$[17]$} & 2016 & Nationwide & $\begin{array}{l}\text { Area socioeconomic } \\
\text { composition of } \\
\text { population in relation } \\
\text { to established clinics }\end{array}$ & $\begin{array}{l}\text { Structure - } \\
\text { establishment of } \\
\text { new practices }\end{array}$ & $\begin{array}{l}\text { New centres located in areas with fewer old adults } \\
\text { living alone and fewer single parents. No significant } \\
\text { effects of income, percentage immigrants, education, } \\
\text { unemployment }\end{array}$ \\
\hline
\end{tabular}

"Grey literature"

Rehnberg et al

Glenngård

[31] 2012 Region Halland, Region Skåne, Region Västra Götaland

Swedish

Association of

Local Authorities

and Regions

Johansson

[30] 2012 Stockholm County Council

Dahlgren et al

[24] 2013 Stockholm County Council

Mohmand

[29] 2014 Stockholm County Council

National Audit

Office

Myndigheten

för vårdanalys

Government investigation "Efficient care" county councils in-depth

[26] 2015 Stockholm County Council, Region Västra Götaland,

[10] 2016 Nationwide
Population register Visits, productivity, data

Patient survey data

Outcome - Patient satisfaction

Survey among 360 PHC managers

Survey among PHC doctors and nurses

Population register data

Interviews with 6 PHC doctors

Population register data, interviews Region Östergötland

Meetings, interviews, documents

Doctors' views on reform and reimbursement systems

Health promotion in $\mathrm{PHC}$ new practices

Process of care doctors' views

Structure establishment of

Population register Process of care PHC visits care, role of PHC
Increase in visits and in productivity overall. Resources decreased in areas with greater need

Satisfaction with primary care higher in areas with low level of social deprivation and in smaller practices

Dissatisfaction with reimbursement systems, leading to prioritization of patients with lesser needs

Negative impact on health promotion because of lack of reimbursement

Visits, patient satisfaction, Increase in visits for all but more among high income earners. Patient satisfaction generally not affected, but lower among patients with greater needs. New practices spread out. new practices

PHC reform makes patients to be customers Reimbursement system prioritises those with lesser needs

More new practices in wealthy larger urban areas, interviews suggesting practitioners not establishing in areas with greater need

Increase in visits among all, stronger among high income earners. Higher increase among person with no diagnosis indicating higher health care need.

Organization of health
PHC very important to the whole health care system, should be first line for all. PHC Choice Reform has made cooperation around patients with complex needs more difficult. Suggest legislation for separate organization of PHC for these patients. 
Table 2 Overview of potential and observed effects of the PHC Choice reform and reimbursement systems on structure, process and outcome in PHC in Sweden

\begin{tabular}{|c|c|c|c|}
\hline & PHC Choice Reform & $\begin{array}{l}\text { Reimbursement system } \\
\text { based on fee-for service }\end{array}$ & $\begin{array}{l}\text { Comments - impact on equity } \\
\text { and need-based care }\end{array}$ \\
\hline \multicolumn{4}{|l|}{ Structure - Access, resources } \\
\hline Number of practices & Increased & & $\begin{array}{l}\text { Less increase in disadvantaged } \\
\text { areas }\end{array}$ \\
\hline Practice distribution & $\begin{array}{l}\text { Providers' choice determines practice } \\
\text { distribution }\end{array}$ & & $\begin{array}{l}\text { Reduced political influence on } \\
\text { distribution by need, may cause } \\
\text { maldistribution }\end{array}$ \\
\hline Resource allocation & $\begin{array}{l}\text { Patients' choice determines resource } \\
\text { allocation between practices }\end{array}$ & $\begin{array}{l}\text { Short visits are incentivised } \\
=\text { more income }\end{array}$ & $\begin{array}{l}\text { Reduced political influence on } \\
\text { resource allocation by need }\end{array}$ \\
\hline GP's work environment & $\begin{array}{l}\text { Patients become customers - change } \\
\text { in professional focus }\end{array}$ & Many short visits are incentivised & $\begin{array}{l}\text { Priority on those with lesser } \\
\text { needs }\end{array}$ \\
\hline \multicolumn{4}{|l|}{ Process - Delivery of health care } \\
\hline Number of visits to PHC & Increased & Increased & $\begin{array}{l}\text { Greater increase for those with } \\
\text { lesser needs }\end{array}$ \\
\hline Prioritisation of patients & Patients as customers & $\begin{array}{l}\text { Focus on short visits by healthier } \\
\text { patients }\end{array}$ & $\begin{array}{l}\text { More demand-driven care. Less } \\
\text { focus on those with greater need }\end{array}$ \\
\hline Integrated care & $\begin{array}{l}\text { More complex to achieve integration, } \\
\text { competition }\end{array}$ & Integrated care not incentivised & $\begin{array}{l}\text { More difficult for those in need } \\
\text { of integrated care }\end{array}$ \\
\hline Holistic care & $\begin{array}{l}\text { De-limited, differentiated PHC assignments } \\
\text { (e.g. ENT, gynaecology, child health) }\end{array}$ & $\begin{array}{l}\text { One visit, one problem (short itemized } \\
\text { visits) }\end{array}$ & $\begin{array}{l}\text { Itemized care not beneficial for } \\
\text { those with complex needs }\end{array}$ \\
\hline Inter-professional care & Focus on doctors & Less teamwork doctors and nurses & $\begin{array}{l}\text { No benefit for those in need of } \\
\text { inter-professional care }\end{array}$ \\
\hline \multicolumn{4}{|l|}{ Outcomes - impact on health } \\
\hline $\begin{array}{l}\text { Health among those with } \\
\text { complex needs }\end{array}$ & $\begin{array}{l}\text { Coordination and integration more } \\
\text { difficult }\end{array}$ & Counteracts holistic care & $\begin{array}{l}\text { Potentially adverse effects on } \\
\text { those with greater needs }\end{array}$ \\
\hline Treatment impact & $\begin{array}{l}\text { Reduced focus on prevention, more } \\
\text { emphasis on cure }\end{array}$ & $\begin{array}{l}\text { Focus on short visits - curative care } \\
\text { for self-limiting diseases }\end{array}$ & $\begin{array}{l}\text { Increase in preventable health } \\
\text { problems? }\end{array}$ \\
\hline Population health & $\begin{array}{l}\text { Focus only on listed individuals limits } \\
\text { population impact }\end{array}$ & $\begin{array}{l}\text { Less emphasis on health promotion } \\
\text { and on collaboration with other } \\
\text { agencies }\end{array}$ & $\begin{array}{l}\text { Reduces PHC impact on } \\
\text { population health }\end{array}$ \\
\hline
\end{tabular}

In contrast, another recent study [17] which adjusted for effects of county council regulation found that PHC clinics established after the PHC Choice Reform were located in areas with fewer older adults living alone as well as fewer single parents, but that no significant effects were noted for mean income, percentage of immigrants, education, unemployment and children $<5$ years. The study concluded that there were some negative effects on geographical equity, but that these were relatively minor [17].

\section{Effects on the reimbursement system and its impact on resource allocation - an example from Stockholm County Council}

An important aspect of the choice reform is that the location of clinics and the patient's choice of provider to a large extent determine the allocation of resources in $\mathrm{PHC}$, as resources follow the patient. In addition, the design of the reimbursement system for PHC may further impact on resource allocation. The design of Swedish reimbursement systems in $\mathrm{PHC}$ vary between different county councils, but most have a mix of capitation (an annual lump sum per listed individual), and fee-forservice (payment per visit), and a smaller portion of payfor-performance (connected to meeting certain set targets) [19]. The capitation part may also be adjusted for need of health care, by taking into account the socioeconomic composition or the burden of disease in the population, which is the case in most county councils. In most county councils the capitation part constitutes most (about 80\%) of the total reimbursement [19].

In terms of reimbursement systems, Stockholm County Council is an outlier and changed its system of resource allocation markedly with the introduction of the reforms. Since the inception of a purchaser/provider model in health care in the 1990s, the county council for many years operated a need-based resource allocation system in order to distribute resources to match the differing needs of health care in the populations of different geographic areas. The system combined need-adjusted capitation (75\%), taking age and socioeconomic composition of the population into account, and fee-for-service (25\%), and 
was in operation until 2007. It resulted in considerable extra resources being allocated to disadvantaged areas, in order to match their greater need of health care [20].

With the implementation of the Choice Reform in Stockholm County Council 2008, this needs-based resource allocation system was abandoned. A new reimbursement system was introduced, with the stated intention of creating equal terms for all providers of PHC (rather than equal terms for patients, as in the previous system). Reimbursement became predominantly based on demand (fee-for-service) (60\%), and partly (40\%) on the number of listed patients in the population (capitation). The capitation was weighted only by age (higher for persons aged 65 years and above) [21]. The notion of creating equal terms for all providers was criticized, as the variation in burden of disease and socioeconomic composition of listed patients creates very different conditions for PHC providers, and would need to be compensated for [22]. The resulting effect on resource allocation was considerable for PHC clinics in disadvantaged areas, one clinic lost more than 30\% of its resources from 1 year to the next. This system was in place from 2008 to 2015, when it was replaced in 2016 by a capitation-dominated (60\%) system, partly weighted for socioeconomic composition.

\section{Effects on the process of PHC - the delivery of PHC Effects on the number of visits}

When assessing the outcomes of the PHC Choice Reform, different outcomes should be considered. One frequently used outcome is change in number of visits. It should be noted, however, that average number of visits to primary care may not be a conceptually sound measure of access to care. First, it is not clear whether an increase in visits is a good or a bad outcome in terms of the health of the population. An increase, for instance, could indicate an increase in morbidity in the population, which the increase in visits may or may not match.

Second, more disadvantaged groups are in greater need of health services because of their greater prevalence of ill-health and poorer recovery. They often have higher rates of primary care visits than their more affluent counterparts, but even so, their higher rates of visits may still not match their higher level of need. Assessments of equity of access by socio-economic status, therefore, have to adjust for the higher health care need of more disadvantaged groups before assessing if access is equitable and whether inequalities in access have changed over time. Only a few studies make this latter adjustment. These two provisos need to be borne in mind when interpreting the findings of the following studies.

A first study from Stockholm County Council in 2008 [21] reported both increased number of visits and productivity in general, and a decrease in resources in areas with greater need.). Another study from the Scania (Skåne) Region [23] found that access to PHC increased in all groups, but particularly among high income earners. Studies from Stockholm county council have shown varying results. Overall there has been a 35\% increase in the number of visits per adult person from 2005 to 2012 [24]. The number of visits per person has increased in all areas, more among high income earners than among low income earners, but more among those with low education than among those with high education [24]. These studies did not adjust for health care needs. However, another study which made this adjustment found that the rate of increase in the number of visits was significantly lower among persons with greater needs (particularly women) and among men born outside Sweden who live in disadvantaged areas [25].

A recent study of three county councils [26] found an increase from 47 to 55\% between 2005 and 2012 in Stockholm county council in the proportion of patients making one or more visits to the doctor. In Region Östergötland the proportion was $47 \%$ over the same time; in Region Västra Götaland the proportion was $55 \%$. There was an increase in the number of visits in all three county councils, most pronounced in Stockholm County Council where the average number of visits was higher than in the other county councils. In general, groups with low education or low income had a relatively higher number of visits than other groups [26] (as would be expected from their higher level of healthcare need), but it is not clear in this study if the higher number of visits in more disadvantaged groups matched their higher level of need.

\section{Effects on the work of doctors and nurses}

Few studies have reported on how the PHC Choice reform and altered reimbursement systems have affected the delivery of PHC, the GP work environment and prioritization of patients. A national survey among 360 public and private PHC managers in Sweden in 2012 [19] found that only $16 \%$ of the respondents considered that the current reimbursement system to a great extent promoted the priorities they wanted to work for. The proportion was higher in county councils where capitation reimbursement was weighted by socioeconomic factors and morbidity. Nine out of ten respondents in Stockholm County Council stated that the reimbursement system incentivized short visits. The proportion of respondents who agreed that the current reimbursement system supported a prioritization of patients with great health care needs was lowest in Stockholm County Council. There were considerable differences in responses between public and private PHC managers. For instance, at a national level, $70 \%$ of public managers agreed with the 
statement that the current principles of reimbursement risks crowding out patients with greater health care needs, compared to nearly $54 \%$ of private managers. Corresponding figures in Stockholm county council were $89 \%$ and nearly $61 \%$, respectively. Nationally, only $20 \%$ of all PHC managers agreed that the current principles of reimbursement support a health promoting and preventive way of work [19].

An interview study with 24 managers of publicly owned PHC centres in Gothenburg in 2013 found that the reform was perceived as a rapid change, enforced through financial incentives, and that prioritization conflicts arise between patient groups with different needs, demands and levels of empowerment [27]. While the average number of visits per patient increased, chronically ill patients were considered to be crowded out by healthier and more verbally demanding patients.

An interview study among district nurses in western Sweden [28] in 2013 found that the focus on economic benefit may limit the cooperation and exchange of experiences within and between different care units. This could in turn have a negative impact on the quality of care due to competition between different care providers. The reimbursement system emphasized many short doctor visits and the role of nurses was played down. Underused resources and restrictions on nurses had a negative impact on their job satisfaction and the working environment, and may have affected the quality of care as a result [28].

Another small in-depth interview study among six GPs in Stockholm County Council in 2013 [29]) distinguished between effects of the PHC Choice Reform, which resulted in patients becoming customers rather than patients, and effects of the fee-for-service reimbursement system, which put the focus on performing many short visits among patients with lesser needs, in order to generate income. The prioritization of patients with lesser needs was perceived not to be in line with the intentions of the Health and Medical Service Act [29].

A study of how the PHC Choice Reform had affected health promotion and prevention in PHC in Stockholm County Council found that financial incentives were geared towards producing many visits, at the expense of health promotion and preventive activities, for which there was not time and no reimbursement [30].

Achieving integrated care for persons with greater health care needs is another goal of $\mathrm{PHC}$ and requires organized collaboration between $\mathrm{PHC}$ and secondary and tertiary health care services, as well as with municipal social services which are responsible for residential care of elderly. A recent government investigation [10] concluded that the PHC Choice Reform has not been conducive, but rather an obstacle, to achieving integrated care. The investigation even suggested that $\mathrm{PHC}$ should be divided into two different organizations: one according to the current PHC Choice Reform and another organization for elderly persons with complex health care needs, which should be exempted from the mandatory PHC choice regulations [10].

\section{Effects on the outcomes of PHC Effects on patient satisfaction}

Patient satisfaction has been investigated in several studies after the PHC Choice Reform. One study [31], based on patient survey data from three county councils (Region Scania, Region Halland and Region Västra Götaland), found that the patient perceived quality was lower in larger cities and in clinics with a greater proportion of the listed having more difficult socioeconomic circumstances, but higher among patients with greater need of health care. Private clinics had higher patient ratings than public clinics, but were to a greater extent located in more affluent areas. Patients at clinics with a greater number of listed patients were less satisfied than those with fewer listed patients [31]. Similar results were shown in another report [32].

\section{Effects on population health}

Before the PHC Choice Reform, the responsibility for health of the population in the catchment area lay with the local PHC clinic. With the reform, the assignment of PHC was limited to the listed individuals, which may have an important impact on the role of PHC in improving public health on a population level. Health promotion may be carried out through collaboration with other local agencies such as schools, social services, employment agency or non-governmental organizations, in working with improving health-related behaviors requiring community-wide action, for instance increasing physical activity or reducing smoking. As this is no longer part of the assignment of $\mathrm{PHC}$ reimbursed by the county council, there is a risk that such collaboration activities are no longer seen as the responsibility of the local PHC clinic, as found in one study in Stockholm County Council [26].

The government investigation which found that the PHC Choice Reform had increased the difficulties in achieving integrated care among elderly with complex needs [10] suggests that PHC is not organized optimally with respect to elderly patients with complex needs.

\section{Discussion}

\section{Effects on the structure of PHC}

In order to follow up the impact of the PHC Choice Reform, different indicators may be studied. In terms of the impact of the PHC Choice Reform on the structure of $\mathrm{PHC}$, it is evident that the reform has increased the number of $\mathrm{PHC}$ clinics and the average number of visits to $\mathrm{PHC}$, but it is debated whether that is a good 
indicator of improvement in access to health care in its wider sense. The evidence reviewed in this paper indicates that increases in number of visits have not been uniform across the population. The National Audit Office concluded that more new clinics had established in already well served areas, and in interviews $\mathrm{PHC}$ providers indicated they were unwilling to establish practices in areas with high levels of need for care, even if reimbursement systems were to take patients' need of care into account (17). However, the study by Isaksson et al concluded that there were some, but only minor, negative effects on equity (16). It is difficult to measure the supply and access to care in correct ways, and on a meaningful area level.

The National Audit Office report suggests that PHC providers have been choosing their patients, rather than patients choosing their $\mathrm{PHC}$ provider, which is actually the reverse of what the PHC Choice Reform was designed to do. If this conclusion holds, it would be an example of Julian Tudor Hart's classical 'inverse care law' about the operation of market forces in health care, as stated in his 1971 seminal Lancet paper [33]: "The availability of health care tends to vary inversely with the need for it in the population. This inverse balance operates more completely where health care is most exposed to market forces, and less so with less exposure."

A crucial impact of the PHC Choice Reform, as evidenced particularly in Stockholm County Council, is a change in resource allocation to the detriment of areas with greater health care needs, through the combined effect of the PHC Choice Reform itself, the fee-forservice dominated reimbursement system without socioeconomically weighted capitation which was in place $2008-2015$, and a difference in health-seeking behavior and demand for health care between different areas.

An illustration is given in Fig. 1, comparing two areas with the same population size, but with different composition of the population with respect to need. If resources are allocated according to need (A), the population in disadvantaged areas will receive more resources than the population in better-off areas. This was the case in the former need-based reimbursement system in Stockholm County Council in place until 2008, in line with the intentions of the Swedish Health and Medical Services Act [1].

However, if resources are distributed only by population size (B), the disadvantaged area will receive less resources in relation to the level of need in that area, and the population in the better-off area will receive more resources in relation to the area's level of need. This situation corresponds to the capitation part of the fee-forservice dominated reimbursement system with no socioeconomic weighting, disregarding population differences in need.

If resources are allocated by demand for health care services $(C)$, the population in the better-off area is likely to get more resources than the population in the disadvantaged area, because their demand for services is greater, in spite of their relatively lesser need of health care services [34]. This situation may represent the effect of the fee-for-service component in the reimbursement system in Stockholm County Council, which generates an increase in the number of visits, as fee-for-service incentivizes many short visits, generated both by demand of patients and by doctors prioritizing such visits.

In this manner, an adverse outcome of the PHC Choice Reform and a demand-oriented reimbursement system, may be a reallocation of resources away from areas with greater need to areas with less health care needs, as evidenced in Stockholm County Council [29].

\section{Effects on process - delivery of PHC}

The evidence suggests that the PHC Choice Reform has increased the number of visits to PHC. However, some reports indicate a greater increase among groups with lesser health care needs than among those with greater health care needs. According to some studies the reform appears to have had a negative impact on the process

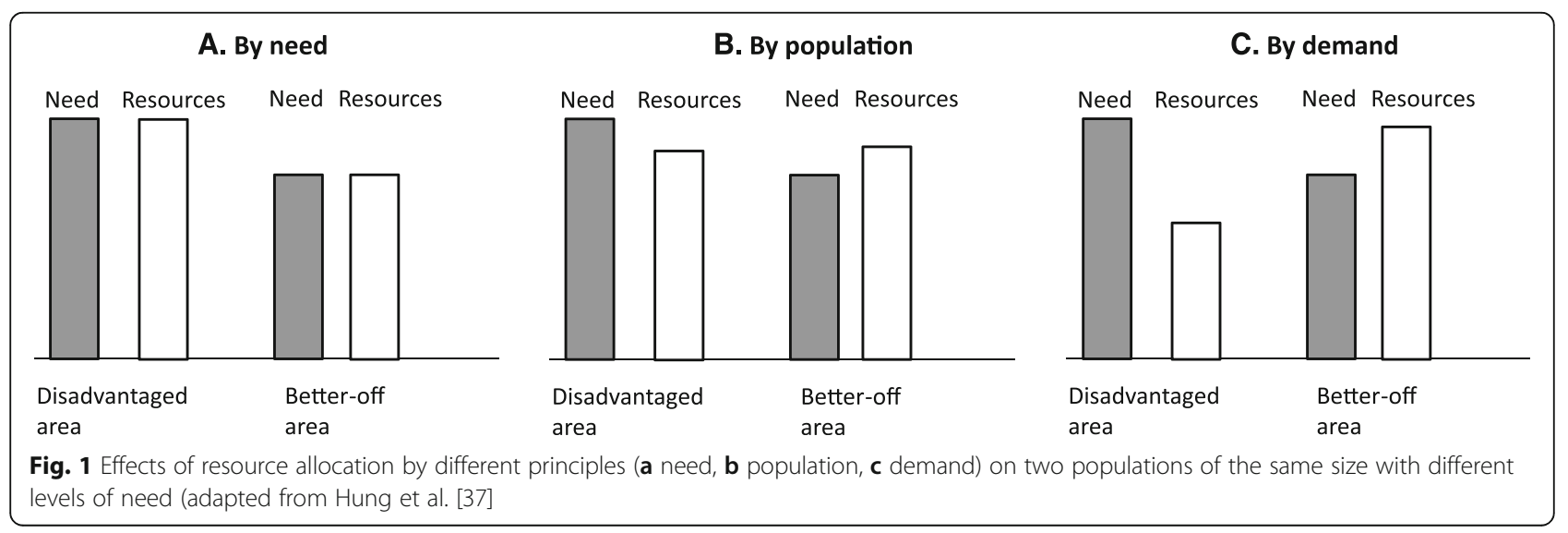


and delivery of PHC, as evidenced by the studies of PHC managers and nurses [19, 23-25]. These studies provide an account of how employees in publicly owned PHC clinics perceive the PHC Choice Reform and changes in reimbursement systems, with a shift in prioritization of patients towards those with lesser needs, and an increased focus on the role of the doctor. As indicated in the national survey of PHC managers, those working in privately owned $\mathrm{PHC}$ clinics were more positive to the reform and changes in reimbursement systems than those working in publicly owned clinics [19]. This is an important aspect to study in further detail.

\section{Effects on outcomes of PHC}

One key finding concerns the impact on integrated care: that the PHC Choice Reform is considered by providers to be an obstacle in organizing integrated care, particularly for elderly with complex needs of care [10]. This is important not least because elderly with complex needs of care are a large and important group of patients in $\mathrm{PHC}$, and the finding is in stark contrast to the intentions of the Health and Medical Services Act [1]. The PHC Choice Reform and change in reimbursement system was also considered by doctors and nurses in Stockholm county council to have reduced the emphasis on health promotion and prevention, because of the focus to produce many visits and generate income to the clinic [26]. The responsibility of PHC for population oriented health promotion activities has also been reduced, because of the focus only on listed patients after the reform, which may further reduce the population health impact of PHC [26].

The interaction between the PHC Choice Reform and simultaneous changes in the reimbursement system provides further difficulties in interpreting the findings of evaluations. The greater increase in the number of visits observed in Stockholm County Council, for example, may be related to the fee-for-service based reimbursement system. In most of the reviewed studies, the number of patient visits to doctors was the measured outcome. However, as this measure depends on the reimbursement system, it may have severe shortcomings. As indicated in some of the referenced studies, the available statistics on the number of visits do not reflect the content or quality or potential effect on health of the visit. As the goal of health care is to improve health, there is a need to go beyond measures of productivity, such as number of visits, to actually measuring the impact on health status improvement, by using patient-reported outcomes [35]. In Stockholm county council there is anecdotal evidence that previously longer visits were divided into several shorter, in order to gain revenue, because of the reimbursement system which gave the same amount for a short as for a longer visit. In some of the studies the increase in number of visits has been greater among those with lesser needs of care [28, 30], in line with an earlier review study of choice reforms in health care in European countries [36].

\section{Conclusion}

In conclusion, the evaluative evidence is sparse and incomplete. The studies to date indicate that the PHC Choice Reform, as implemented by some county councils, predominantly Stockholm, increased access to PHC and increased the average number of visits to PHC, but seems to have particularly benefitted those in more affluent groups and with lower health care needs. In addition, it has made integrated care for those with complex needs more difficult. Among GPs and nurses in PHC there are mixed opinions about the reform. Some consider that persons with greater needs are not prioritized; others are more positive. Resource allocation to PHC has become more dependent on provider location, patient choice and demand, and less on need of care. In view of the more restricted assignment there is also a risk of a reduced impact on population health of PHC. On the available evidence, the PHC Choice Reform may have damaged equity of primary health care provision, contrary to the tenets of the Swedish Health and Medical Service Act. This situation needs to be carefully monitored and countered where necessary. Further studies are needed to follow up the long-term impacts of the reform on the structure, process and outcomes of PHC in Sweden and how different types of reimbursement systems may moderate these impacts.

\section{Abbreviations \\ GP: General Practitioner; PHC: Primary Health Care}

\section{Acknowledgements}

We acknowledge comments on previous versions of the manuscript by Janne Agerholm, Karin Josefsson and Sofie Vengberg. We are grateful for comments by anonymous reviewers.

\section{Funding}

This study was partly supported by a research grant (2014-4763) from the Swedish Research Council for Health, Working Life and Welfare.

\section{Availability of data and materials}

Data sharing not applicable to this article as no datasets were generated or analysed during the current study.

\section{Authors' contributions}

$\mathrm{BB}$ reviewed the articles and reports quoted in the article and wrote the first and final drafts of the article. KB, GN, GT, MW and UW discussed the findings, critically reviewed and revised previous drafts of the article and approved the final draft. All authors read and approved the final manuscript.

\section{Competing interests}

The authors declare that they have no competing interests.

\section{Consent for publication}

Not applicable.

Ethics approval

This study was a review and did not require ethics approval. 


\section{Author details}

${ }^{1}$ Department of Public Health Sciences, Equity and Health Policy Research Group, Karolinska Institutet, SE 17177 Stockholm, Sweden. ²Department of Learning, Informatics, Management and Ethics, Health Outcomes and Economic Evaluation Research Group, Karolinska Institutet, Stockholm, Sweden. ${ }^{3}$ Department of Neurobiology, Care Sciences and Society, Karolinska Institutet, Stockholm, Sweden. ${ }^{4}$ Department of Learning, Informatics, Management and Ethics, Karolinska Institutet, Stockholm, Sweden. ${ }^{5}$ Department of Public Health and Society, Institute of Psychology, Health and Society University of Liverpool, Liverpool, UK. ${ }^{6}$ Department of Public Health and Caring Sciences, Health Services Research, Uppsala University, Uppsala, Sweden.

Received: 21 September 2016 Accepted: 20 January 2017

Published online: 28 January 2017

\section{References}

1. Macinko J, Starfield B, Shi L. The contribution of primary care systems to health outcomes within OECD countries 1970-1998. Health Serv Res. 2003;38:831-65.

2. Hälso- och sjukvårdslagen [Health and Medical Services Act]. SFS 1982:763. (In Swedish)

3. Public Health Agency of Sweden. Folkhälsan i Sverige. Årsrapport 2014 [Public health in Sweden. Annual Report 2014]. https://www. folkhalsomyndigheten.se/pagefiles/17825/Folkhalsan-i-Sverige-arsrapport-2014. pdf. Accessed 4 Mar 2016 (In Swedish)

4. Socialdepartementet. Commission on Equity in Health. http:// kommissionjamlikhalsa.se/en/home. Accessed 4 Mar 2016

5. Anell A, Glenngård AH, Merkur S. Health systems in transition - Sweden: Health system review. Health Systems Transit. 2012;14(5):1-159.

6. Blomqvist P, Winblad U. Sweden: Continued Marketization within a Universalist System. In: Pavolini E \& Guillén A.M. (eds.). Health Care Systems in Europe under Austerity: Institutional Reforms and Performance. Palgrave Macmillan Publishers, 2013

7. Esping-Andersen G. The three worlds of welfare capitalism. Polity Press, 1990.

8. Rothstein B. Vad bör staten göra? Om välfärdsstatens moraliska och politiska logik [What should the state do?] Stockholm: SNS Förlag, 2004. (In Swedish)

9. OECD. OECD health data 2015. http://www.oecd-ilibrary.org/social-issues migration-health/data/oecd-health-statistics/oecd-health-data-health-careutilisation data-00542-en. Accessed 26 Jan 2017

10. Regeringen. Effektiv vård [Effective/efficient care]. SOU 2016:2. Government investigation.

11. Government of Sweden. Lag om valfrihetssystem (Freedom of Choice Act) 2008/09:29. Stockholm: Ministry of Social Affairs, Government of Sweden, 2008. (In Swedish)

12. Ekman B, Wilkens J. A literature review of the regional implementation of the Swedish government's health care reforms on choice and privatization. Heal Econ Rev. 2015:5:39.

13. Anell A. Choice and privatisation in Swedish primary care. Health Econ Policy Law. 2011;6:549-69.

14. Maynard A. Beware of the libertarian wolf in the clothing of the egalitarian sheep: an essay on the need to clarify ends and means. In: Mclntyre D, Mooney G, editors. The economics of health equity. Cambridge: Cambridge University Press; 2007.

15. Fredriksson M, Blomqvist $P$, Winblad U. The trade-off between choice and equity: Swedish policymakers' arguments when introducing patient choice J European Social Policy. 2012;23:192-209.

16. Donabedian A. The quality of care - how can it be assessed? JAMA. 1988:260:1743-8.

17. Isaksson D, Blomqvist $P$, Winblad U. Free establishment of primary care providers: effects on geographical equity. BMC Health Serv Res. 2016;16:28.

18. Riksrevisionen [National Audit Office]. Primärvårdens styrning - efter behov eller efterfrågan? [Primary health care governance - based on need or demand?]. Stockholm: Riksrevisionen, 2014. (RiR 2014:22). (In Swedish)

19. Swedish Association of Local Authorities and Regions (SKL). Vårdval i primärvården [Patient choice in primary care]. http://webbutik.skl.se/ bilder/artiklar/pdf/7164-826-6.pdf?issuusl=ignore. Accessed 20 Dec 2012 (In Swedish)
20. Stockholm county council. Behovsindex 2011-2013 [Care need index 20112013]. http://dok.slso.sll.se/CES/FHG/Jamlik_halsa/Rapporter/Behovsindex2011-2013.pdf. Accessed 10 Sept 2016 (In Swedish)

21. Rehnberg C, Janlöv N, Khan J. Uppfölining av Vårdval år 2008 [Follow-up of PHC Choice Reform Stockholm 2008]. http://dok.slso.sll.se/CES/FHG/Jamlik_ halsa/Rapporter/Aldre_rapporter/vardval-stockholm-uppfoljning.2009_6.pdf. Accessed 10 Sept 2016 (In Swedish)

22. Halldin J. Vårdval Stockholm skapar en orättfärdig och ojämlik vård [Stockholm PHC Choice Reform creates inequitable care]. http://www. lakartidningen.se/Functions/OldArticleView.aspx?articleld=13558. Accessed 10 Sept 2016 (In Swedish)

23. Beckman A, Anell A. Changes in health care utilisation following a reform involving choice and privatisation in Swedish primary care: a five-year follow-up of GP-visits. BMC Health Serv Res. 2013;13:452.

24. Dahlgren $\mathrm{C}$, Brorsson $\mathrm{H}$, Svereus $\mathrm{S}$, Rehnberg C. Fem år med husläkarsystemet inom Vårdval Stockholm [Five years with choice in primary care in Stockholm]. Stockholm: Karolinska institutet; 2013 (Report), . (In Swedish).

25. Agerholm J, Bruce D, Ponce de Leon A, Burström B. Equity impact of a choice reform and change in reimbursement system in primary care in Stockholm County Council. BMC Health Serv Res. 2015;15:420. doi:10.1186/s12913-015-1105-8.

26. Myndigheten för Vårdanalys. Vårdval och jämlik vård inom primärvården. En jämförande studie mellan tre landsting före och efter vårdvalets införande. [PHC Choice Reform and equitable care in primary health care. A comparative study of three county councils.] Stockholm: Myndigheten för vårdanalys, 2015. (Rapport 2015:6) (In Swedish)

27. Maun A, Nilsson K, Furåker C, Thorn J. Primary healthcare in transition - a qualitative study of how managers perceived a system change. BMC Health Serv Res. 2013;13:382.

28. Hollman D, Lennartsson S, Rosengren K. District nurses' experiences with the freechoice system in Swedish primary care. Br J Community Nurs. 2014;19:30-5.

29. Mohmand S. Vård på lika villkor i primärvården efter vårdval i Stockholm [Equitable care in primary care after the choice reform in Stockholm]. En kvalitativ studie. Examensarbete, läkarprogrammet, Karolinska institutet Stockholm, 2014. (In Swedish)

30. Johansson H. Vårdval Stockholm, Husläkarverksamheten och det hälsofrämjande arbetet. En studie med utgångspunkt från vårdens professioner [Choice in PHC in Stockholm: PHC and health promoting work]. Umeå: Umeå universitet, Folkhälsa och klinisk medicin/ epidemiologi och global hälsa, 2012. (In Swedish)

31. Glenngård AH. Vad påverkar patientupplevd kvalitet i primärvården? [What affects patient perceived quality of care in primary care?]. Stockholm: Myndigheten för vårdanalys, 2012. http://www.vardanalys.se/Global/ Rapporter\%20pdf-filer/2012/R1_2012_Vad_paverkar_patientupplevd.pdf. Accessed 10 Sept 2016 (In Swedish)

32. Glenngård $\mathrm{AH}$. Is patient satisfaction in primary care dependent on structural and organizational characteristics among providers? Findings based on data from the national patient survey in Sweden. Health Econ Policy Law. 2013;8:317-33.

33. Hart JT. The inverse care law. Lancet. 1971:297:405-12.

34. Burström B. Market-oriented, demand-driven health care reforms and equity in health and health care utilization in Sweden. Int J Health Serv. 2009:39:271-85.

35. Appleby J, Devlin N, Parkin D. Using patient reported outcomes to improve health care. Oxford: Wiley Blackwell; 2016.

36. Fotaki $M$, Roland $M$, Boyd $A$, McDonald $R$, Scheaff $R$, Smith $L$, Fotaki M, Roland M, Boyd A, McDonald R, Scheaff R, Smith L. What benefits will choice bring to patients? Literature review and assessment of implications. J Health Serv Res Policy. 2008;13:178-84. doi:10.1258/ jhsrp.2008.007163.

37. Hung PM, Dzung TV, Dahlgren G, Tuan T. Vietnam: Efficient, equity-oriented financial strategies for health. In: Evans T, Whitehead M, Diderichsen $\mathrm{F}_{\text {, }}$ Bhuiya A, Wirth M, editors. Challenging inequities in health. From ethics to action. Oxford: Oxford University Press; 2001. p. 296-306. 